\title{
THE TIME DOMAIN ANALYSIS OF INTERACTIONS IN THE WHEEL - RAIL CONTACTS DUE TO DISCONTINUOUS TIME PERIODIC LOADS
}

\author{
Maria Trzaska \\ Institute of Precision Mechanics \\ Duchnicka Street 3, 01-796 Warsaw, Poland \\ tel.: +4822 5602902, fax: +48226634332 \\ e-mail: maria.trzaska@imp.edu.pl \\ Zdzisław Trzaska \\ Warsaw University of Ecology and Management \\ Department of Management \\ Wawelska Street 14, 02-061 Warsaw, Poland \\ tel.: +4822 8258035, fax: +48228258031 \\ e-mail: zdzislaw.trzaska@netlandia.pl
}

\begin{abstract}
In this paper, we present Fourier series less approaches for determining the energy produced by discontinuous time periodic forces in the wheel-rail contacts of the railway track systems. The vehicle system model used in this study consists of a quarter car supported on a bogie, while the side frame is supported on two wheel sets. The main advantage of the presented method is the total elimination of frequency analysis, which in effect introduces important simplifications in the identification of the energetic effects in the contact. Fundamental properties of contact displacements of the rail surface have been considered on the basis of the newly established method. The contact zone between railway wheels and the rail surfaces made of bulk materials is perceived as strong enough to resist the normal (vertical) forces introduced by heavy loads and the dynamic response induced by track and wheel irregularities. The analysis is carried out for a wheel running on an elastic rail rested on sleepers arranged on completely rigid foundation. The established model of the wheel-rail contact dynamics has been applied to that same roll plane, but with taking into account a nonlinear characteristic of the sleeper with respect to the ground. Attention then is focused completely on the modelling of the energy absorbed by the rail. The applied method employs the energy state variables as time functions leading to determine the susceptibility of a given contact on the strength induced by the rail roll.
\end{abstract}

Keywords: railway transport, wheel-rail contact, discontinuous time periodic forces, energy absorbed by the rail, strength susceptibility

\section{Introduction}

Contact forces between a wheel and a rail vary dynamically during the passage of a wheel on the surface of the rail. A discontinuous variation of contact forces causes undesired deteriorations of a track and its substructures. Therefore, these dynamic contact forces are of main concern of the railway engineering. Increasing operational speeds and comfort demands require paying attention on both riding qualities of railway vehicles, transport safety and elimination of negative effects on the environment. As the challenges of higher speed and higher loads with very high levels of safety require ever more innovative engineering solutions, better understanding of the technical issues and use of new computer based tools appear as very important and needed activities $[2,3,5]$.

Accurate and relevant analysis of track system dynamics is necessary in order to determine the response of new system designs, as well as to predict the effects of proposed modifications on 
responses of existing systems or to determine the modifications essential to enable a system to give the required response. It should be emphasized that in periodic non-sinusoidal states even relatively small track systems may exhibit fairly complex dynamical behaviour. The traditional tools for analysis of periodic non-sinusoidal waveforms are the Fourier series and/or fast Fourier transforms (FFT) as well as the sampling theorem of Shannon, Whittaker and Kotel'nikov [4]. It is worth to mention that a discontinuous signal, like the square wave, cannot be expressed as a sum, even an infinite one, of continuous signals. The extraneous peaks in the square wave's Fourier series never disappear; they occur whenever the signal is discontinuous, and will always be present whenever the signal has jump discontinuities. The main theorem concerning the convergence of the Fourier series at a discontinuity implies that this series converges to $f(t)$ except at the point $t=t_{0}$, which is a point of discontinuity of $f(t)$ [10]. Indeed, Gibbs [13] showed that if $f(t)$ is piecewise smooth on $[0, T]$ and $t_{0}$ is a point of discontinuity, then the Fourier partial sums would exhibit the same behaviour, with the bump's height almost equal to

$$
\Delta f\left(t_{0}\right)=0.18\left[f\left(t_{0}^{+}\right)-f\left(t_{0}^{-}\right)\right] .
$$

Recall that the notations $f\left(t_{0}^{+}\right)$and $f\left(t_{0}^{-}\right)$represent the right-limit and left-limit, respectively, of $f(t)$ at the point $t_{0}$.

Thus, it is evident that more formal time-domain mathematical tools are needed for accurate analysis of large systems and complicated harmonic producing elements. The time domain representation of a track system by means of the concatenation procedure, as opposed to the frequency domain representation by means of the system transfer function, became the more advantageous approach to the exhibition of system dynamics in periodic non-harmonic states. Especially, the introduction of geometric tools like hysteretic loops on energy phase plane greatly advances the theory and enables the proper generalization of many fundamental concepts known for computer aided geometric designs to the world of periodic non-harmonic waveforms.

The purpose of this paper is to demonstrate guidelines for the preparation and analysis of periodic non-harmonic problems through case studies and simulation examples. The presented method is very straightforward and appears as a powerful broadly applicable technique that enables us to characterize non-harmonic periodic oscillations from a perspective different than that obtained by the method resulting from the Fourier series applications. Resulting illustration procedures are emphasized using Matlab mfiles [8].

The paper is organized as follows. Next section is devoted to preliminary formulations and is focused on inconveniences following from applications of Fourier series to analysis of complex dynamical system operating in periodic non-sinusoidal conditions. Concatenation procedure is established in section three. Problems concerning the wheel-rail contact dynamics are presented in section four. Section five is devoted to hysteretic loops of one-period energy and rail's material behaviour is characterized in section six. Summary and conclusions are included into section seven.

\section{Representation of discontinuous time periodic waveforms}

To avoid the difficulty appearing in practical applications of the Fourier series approach to obtain exact solutions of problems involved by periodic non-sinusoidal excitations in track systems we propose to use a so-called carrying periodic waveform defined as follows

$$
p(t)=p(t+T)=\frac{T}{2}-\frac{T}{\pi} a \tan \left(\cot \frac{\pi}{T} t\right),
$$

where $T$ denotes the period. The direct plot of (2) for $T=\pi[\mathrm{s}]$ is presented in Fig. 1a.

In the sequel we will also take advantages shifted Heaviside step function: 


$$
H(t, \tau)=\frac{1}{2}\left(1+\frac{a b s(t-\tau)}{t-\tau}\right)= \begin{cases}0 & \text { for } \mathrm{t} \leq \tau^{-} \\ 1 & \text { for } \mathrm{t}>\tau^{+}\end{cases}
$$
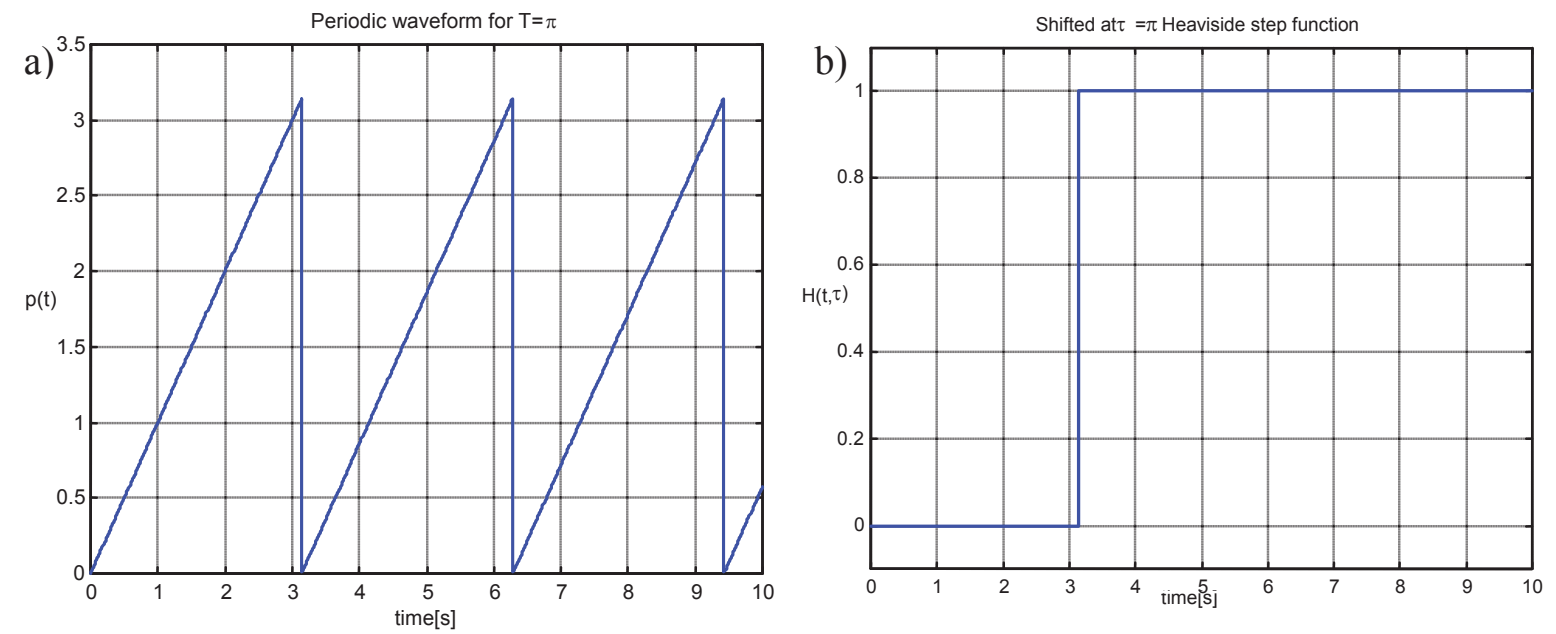

Fig. 1. Waveforms: a) $p(t, \pi), b) H(t, \pi)$

The plot of (3) for $\tau=\pi[\mathrm{s}]$ is presented in Fig.1b. The counterpart of (3) for $p(t)$, e.g. $H(p(t), \tau)$ for $T=5.5 \mathrm{~s}$ and $\tau=\pi[\mathrm{s}]$ is plotted in Fig.2a. Using the carrying periodic waveform $p(t)$ and periodic shifted Heaviside step function $H(p(t), \tau)$ we can easily determine the waveform $g(t)=g(t+2 \pi)$ with $g(t)=-\sin (2 t)$ for $0 \leq t \leq \pi$ and $g(t)=\sin (2 t)$ for $\pi \leq t \leq 2 \pi$ e.g. we will to obtain the waveform $g(t)=\sin (2 p(t)) r(p(t), \pi)$ for $0 \leq t \leq 2 \pi$, where $r(t)=2 H(p(t, \tau))-1$. It is shown in Fig. $2 \mathrm{~b}$.
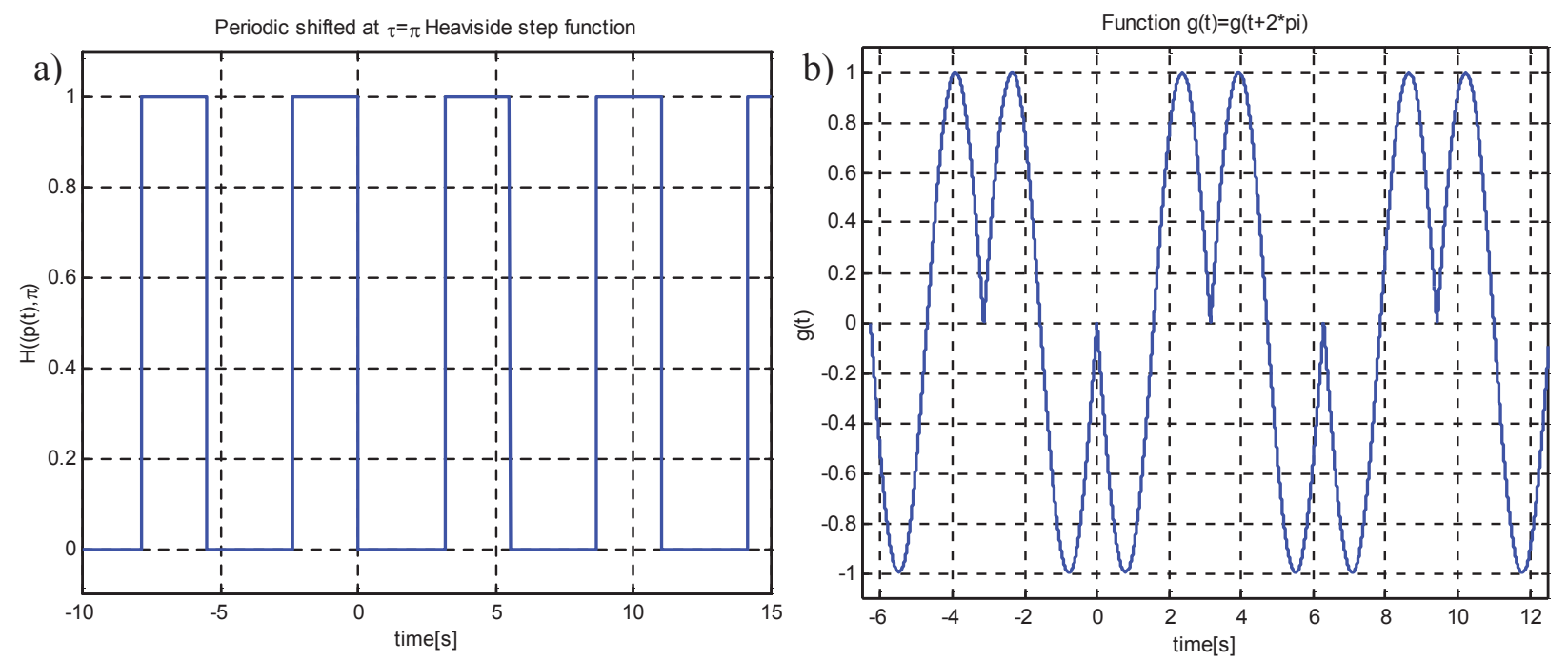

Fig.2. Periodic waveforms: a) shifted Heaviside step function, b) waveform composed of sinusoidal terms

The above waveforms and functions as well as many other similar ones derived on their base are very useful in relatively simple representations of composite periodic non-harmonic both continuous as well as discontinuous waveforms.

\section{Straightforward method for periodic non-harmonic states in linear systems}

The demand made on many present day track systems is so severe, that the analysis and assessments of their dynamic performance is now an essential and very important part of system design. Dynamic analysis must be performed so that the system response to the expected excitation can be predicted, and modifications made as necessary. It is also an essential technique to 
apply to existing dynamic track systems, when considering the effects of modifications and searching for performance improvement. There have been very many cases of systems failing or not meeting performance targets because of oscillation fatigues or movement excessive amplitudes of one component or another. Because of the very serious effects, which unwanted oscillations can have on dynamic systems, it is essential that oscillation analysis must be carried out as an inherent part of their design, when necessary modifications can most easily be made to eliminate unwanted oscillations or at least to reduce their amplitudes as much as possible.

In this Section we concentrate our attention on some general ideas associated with periodic non-harmonic solutions and to those topics that arise in a more valuable improving of studies of oscillatory track systems. The attention is focused on second-order linear systems since they form the basic structure for the posing and analysing of a broad spectrum of problems in track systems.

It is well known that a discontinuous signal, like the square wave, cannot be expressed as a sum, even with an infinite number of terms, of continuous signals. Quite obviously, if the forcing waveform is subject to jump changes the linear smoothing procedure is not a good choice anymore, because all linear systems confuse and remove the high frequency components from the output waveforms. For this reason, when a source waveform with jumps is applied to a linear system it causes a typical effect of "edge blurring".

Taking into account the above requirements and insufficiencies of the methods based on Fourier series, which are up-to-date most commonly, used for studies of periodic non-harmonic states of track systems we propose in the sequel new method for obtaining, in closed form, the response of any linear system corresponding to piecewise-continuous periodic non-harmonic forcing terms. In our approach, the solution is exact, and by means of suitable unification of its piecewise representation, we can get with ease the exact expressions for its time derivatives. The method presented here depends on the carrying periodic waveform and of a concatenation procedure for the unified representation of composite periodic non-harmonic waveforms. We discus properties of linear systems with periodic non-harmonic excitations and develop a systematic Fourier series-less method for their studies.

To cope with the undesirable effects we will describe discontinuous waveforms by using the carrying periodic waveform and its relatives such as switch-on and switch-of waveforms. Thus the waveform shown in Fig. 3 is represented as:

$$
g(t)=g_{1}(t)+H_{1}\left(t, t_{1}\right)\left[g_{2}(t)-g_{1}(t)\right]+H_{2}\left(t, t_{2}\right)\left[g_{3}(t)-g_{2}(t)\right] .
$$

This intuitively appealing "switching rule" can be exploited in several ways. Using the switching.

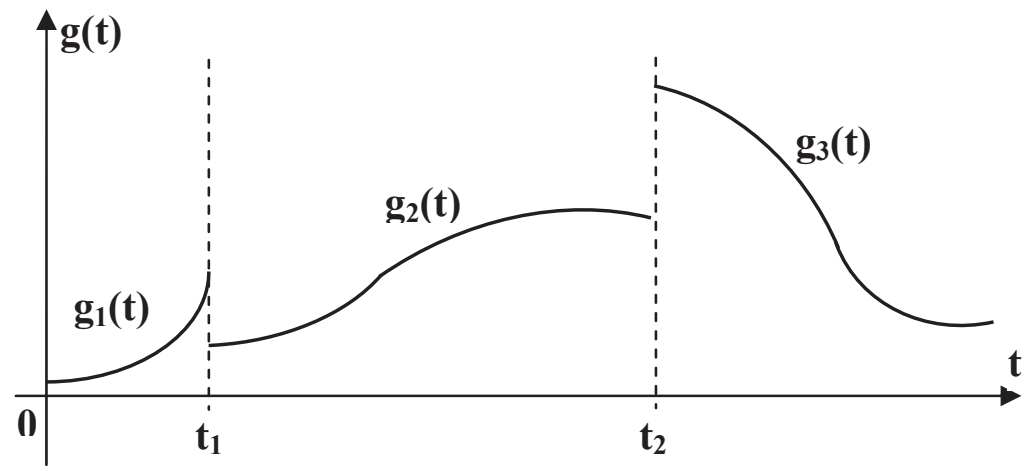

Fig. 3. Illustration of discontinuities of a forcing waveform

approach (4) suggests the incorporation of a true smoothing element into the competition. From the standpoint of other approaches that pursue the same target, basically there is a new concept regarding the use of powerful developments. One of the advantages of introducing this additional complexity into the system is the ability to combine useful properties of each of the complex structures of the load. 
To present the main idea of our approach we consider at first a linear system described by the following equation:

$$
\ddot{x}(t)+2 \dot{x}(t)+4 x(t)=u(t) .
$$
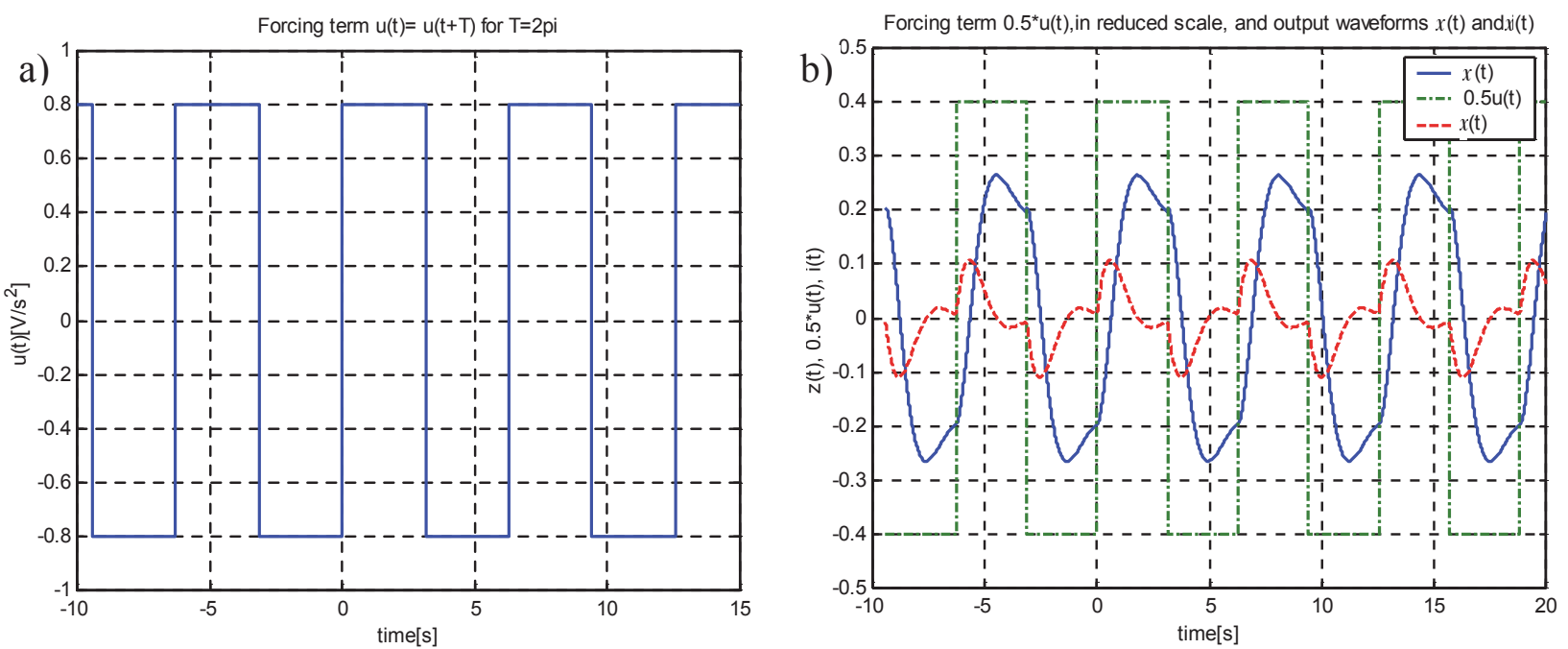

Fig. 4. Steady-state waveforms: a) forcing term, b) output terms

where the waveforms of the system forcing term and the system response are denoted by $u(t)$ and $x(t)$, respectively. Let the forcing term takes the form shown in Fig. 4a. This means that in the $p(t)$ variable domain we have $u(t)=0.8 r(t)$.

In this case the self-frequencies of the system are as follows

$$
s_{1}=-1+j \sqrt{3}, \quad s_{2}=-1-j \sqrt{3} .
$$

The steady-state solutions for the periodic output waveform in successive semi-periods of the forcing waveform take the forms:

- for $0 \leq t \leq \pi$

$$
x_{1}(t)=0.2+e^{-t}\left[A_{1} \cos (\sqrt{3} t)+B_{1} \sin (\sqrt{3} t)\right]
$$

- for $\pi \leq t \leq 2 \pi$

$$
x_{2}(t)=-0.2+e^{-t}\left[A_{2} \cos (\sqrt{3} t)+B_{2} \sin (\sqrt{3} t)\right],
$$

where the integration constants $A_{1}, B_{1}, A_{2}$ and $B_{2}$ are to be determined from the respective conditions for the periodicity and analytical continuity of the total solution $x(t)$.

Taking into account the periodicity and analytical continuity conditions we get:

$$
x_{1}(0)=x_{2}(2 \pi), \quad \dot{\mathrm{x}}_{1}(0)=\dot{x}_{2}(2 \pi)
$$

and

$$
x_{1}(\pi)=x_{2}(\pi), \quad \dot{\mathrm{x}}_{1}(\pi)=\dot{x}_{2}(\pi) .
$$

Solving the above equalities with respect to the integration constants yields

$$
A_{1}=-0.3953, A_{2}=9.9876, B_{1}=-0.2117, B_{2}=-3.6316 \text {. }
$$

Substituting these values into (7) and (8) and mapping the solution of (5) into the $p=p(t)$ domain gives:

$$
\begin{aligned}
& x(t)=x_{1}(p)+H(p, \pi)\left[x_{2}(p)-x_{1}(p)\right] \\
& \quad=-0.2 r(p)+e^{-p}\{[4.7988+5.1941 r(p)] \cos (\sqrt{3} p)+[-1.9177-1.706 r(p)] \sin (\sqrt{3} p)\} .
\end{aligned}
$$


Figure $4 \mathrm{~b}$ represents the forcing waveform $u(t)$, in twice-reduced scale, the output waveforms of the displacement $x(t)$ and the velocity $\dot{x}(t)$.

The above result can be easily generalized for system representations by a standard state variable equation:

$$
\dot{\boldsymbol{x}}(t)=\boldsymbol{A}_{1} \mathbf{x}(t)+\mathbf{g}(t),
$$

where vectors $\boldsymbol{x}(t)$ and $\boldsymbol{g}(t)$ as well as constant element matrix $\boldsymbol{A}_{1}$ have appropriate dimensions. Here it is worth noticing that $\boldsymbol{A}_{1}$ must be non-critical with respect to $T$, i.e. the relation:

$$
\operatorname{det}\left(\mathbf{I}-e^{A_{1} T}\right) \neq 0
$$

must be fulfilled.

The discontinuous forcing term $\boldsymbol{g}(t)=\boldsymbol{g}(t+T)$ can be represented by introducing the unit Heaviside step functions $H_{k}\left(t, T_{k}\right), k=1,2, \ldots n$ being shifted at the portions of the period with respect to the origin point $t=0$, namely:

$$
\boldsymbol{g}(t)=\boldsymbol{g}_{1}(t)+\sum_{k=1}^{n} H\left(t, T_{k}\right)\left[\boldsymbol{g}_{k+1}(t)-\boldsymbol{g}_{k}(t)\right],
$$

where $\boldsymbol{g}_{k}(t), k=1,2, \ldots, n$ denotes $k$-th continuous term of the applied force in the subintervals of $[0, T)$. Such a division yields the respective solutions $\boldsymbol{x}_{k}(t)$ of $\boldsymbol{x}(t)$ that are determined as:

$$
\mathbf{x}_{k}(t)=e^{\mathbf{A}_{1} t} \mathbf{J}_{k}+\boldsymbol{x}_{f, k}(t),
$$

for $k=1,2, \ldots, n$, where $\boldsymbol{x}_{f, k}(t)$ is a forced steady state (index $f$ ) solution of (10) and has the same waveform shape as that of $\boldsymbol{g}_{k}(\mathrm{t})$ in the corresponding subinterval, and $\boldsymbol{J}_{k}$ is a constant vector to be determined. We can find vectors $\boldsymbol{J}_{k}$ by analyzing the periodicity condition for the total solution as follows:

$$
\mathbf{J}_{1}+\mathbf{x}_{f, 1}(0)=e^{\mathbf{A}_{1} T} \mathbf{J}_{n},
$$

and from the continuity conditions it follows that:

$$
e^{\mathbf{A}_{1} T_{k}} \mathbf{J}_{k}+\boldsymbol{x}_{f, k}\left(T_{k}\right)=e^{\mathbf{A}_{1} T_{k}} \mathbf{J}_{k+1}+\boldsymbol{x}_{f, k+1}\left(T_{k}\right),
$$

for $k=1,2, \ldots, n$.

Because the period $[0, T)$ is divided into subintervals $\left[t_{k}, t_{k+1}\right), k=1,2, \ldots, n$, then (14), (15) yield the block matrix equation:

$$
\left[\begin{array}{cccc}
\boldsymbol{I} & \mathbf{0} & \cdots & -e^{\boldsymbol{A}_{1} T} \\
e^{A_{1} T_{1}} & -e^{A_{1} T_{1}} & \cdots & \mathbf{0} \\
\vdots & \vdots & \vdots & \vdots \\
\mathbf{0} & \mathbf{0} & \cdots & -e^{A_{1} T_{n}}
\end{array}\right]\left[\begin{array}{c}
\boldsymbol{J}_{1} \\
\boldsymbol{J}_{2} \\
\vdots \\
\boldsymbol{J}_{n}
\end{array}\right]=\left[\begin{array}{c}
\boldsymbol{x}_{f, 1}(T)-\boldsymbol{x}_{f, 1}(0) \\
\boldsymbol{x}_{f, 2}\left(T_{1}\right)-\boldsymbol{x}_{f, 1}\left(T_{1}\right) \\
\vdots \\
\boldsymbol{x}_{f, n-1}\left(T_{n}\right)-\boldsymbol{x}_{f, n}\left(T_{n}\right)
\end{array}\right]
$$

From the Kronecker-Weierstrass form [13] it follows that the system (16) has a unique solution $\left\{\boldsymbol{J}_{1}, \ldots, \boldsymbol{J}_{n}\right\}$ for any $T, T_{1}, \ldots, T_{n}$, and $\boldsymbol{A}_{1}$. Using the carrying periodic waveform $p(t)$ we sew on continuous solutions in all subintervals during the concatenation process yielding periodic steady state solutions. Because the solutions are exact, the typical drawbacks of classical methods, such as the Gibbs effect [4], are avoided

\section{Dynamic model of wheel-rail contacts}

With the significant increase of train speed and axle load, the vibration of the coupled vehicle and track system due to corrugations and spoils of rails and wheels is presently strongly intensified 
and the safe operation of trains is reduced. A further problem arises when the wheel-rail contact is subject to an action of time-discontinuous forces. It is well known that in the contact zone between railway wheel and rail the surfaces and bulk material must be strong enough to resist the normal (vertical) forces introduced by heavy loads and the dynamic response induced by track and wheel irregularities. The interactions of the wheel surface and the volume of a solid rail are very important [2]. Several modelling methods can be applied and in the course of the last decade new demands emerged in the analysis of wheel-rail contact. They are mainly connected with the reduction of costs of maintenance, technical diagnostics of the track and of railway vehicles, and elimination of negative effects on the environment $[1,3,5]$.

In this section two-dimensional model of the wheel-rail pair of the wheelset is taken into account upon consideration of three subsequent layers. These include the rail, and the lumped representation of the sleeper and the ballast. The rail is discretely supported on the sleepers, and together ballasts/subballasts with subgrades elements, as shown in Fig. 5a, where two layers of discrete masses represent the rail and the sleeper with the ballast, respectively. The rail is not considered a Timoshenko beam, but as a result of ordinary beam theory - i.e. Bernoulli-Euler theory - with uniform flexible rigidity and added mechanisms effectively lowering the stiffness of the beam. Therefore, it can be divided into small segments so that a sleeper is assigned to every segment. The sleeper has both a mass and the pad between rail and sleeper and the ballast are replaced by springs and dampers. As a natural improvement a more complex model can also be considered. It must take into account the roll effect of the other wheel - rail pair of the wheel set. In light of this reason it is assumed for the developments that follow that the motion of each wheelset tracks follows exactly the geometry of the railway and lateral inclinations can simply be ignored during numerical simulation $[5,10]$.

The rail structure is particularly subjected to dynamic load, which is induced by moving wheels of the track vehicles. When an elastic body, such as a wheel, is pressed against another elastic body, such as a rail, so that a normal load is transmitted and a contact area is formed. As the elastic deformation in the vicinity of the contact area is small its effect on the stress response cannot be neglected. Then, assuming that in the vicinity of the contact patch the curvatures of the wheel and rail are constant, the imprinted contact patch is small compared with the radii of curvature and the dimensions of the wheel and rails, the contacting bodies can be represented by elastic half-spaces and their shape can be approximated by quadratic surfaces [7]. The equation of motion of a beam is given by the 4 th-order partial differential equation:

$$
E I \frac{\partial^{4} z(x, t)}{\partial x^{4}}+M_{r} \frac{\partial^{2} z(x, t)}{\partial t^{2}}=F(t) \delta\left(x-x_{s}\right) .
$$

where the coordinate $x$ represents the longitudinal position of the beam with respect to the left end support of the rail beam, $x_{s}$ defines the position of the $s$ th sleeper, $E$ is the elastic modulus of rail beam materials and $I$ is the second moment of area, $\delta(x)$ is the Dirac delta impulse and $F(t)$ is force developed at the $s$ th rail/sleeper interface.

Applying the finite difference method, a flexural deformation of the rail is expressed using the local coordinate $y=x / h$ and the equation of motion of the entire system is obtained as:

$$
\boldsymbol{M} \ddot{z}(t)+\boldsymbol{B} \dot{\boldsymbol{z}}(t)+\boldsymbol{K} \boldsymbol{z}(t)=\boldsymbol{F}(t),
$$

where $\boldsymbol{M}, \boldsymbol{B}, \boldsymbol{K}$ and $\boldsymbol{F}(t)$ are the mass, damping, rigidity matrix and forcing vector, respectively.

Usually it can be assumed that the material properties of wheel and rail are the same and in this case it can be shown that the tangential tractions do not affect the normal pressures acting between the bodies. Then with these assumptions, for the case where the wheels and rails are smooth, the dimensions of the contact area can be obtained from the theory of Kalker described in [9]. For each of such segments as that shown in Fig. 5c a system of equations can be formulated as follows: 


$$
\left[\begin{array}{cc}
M_{r} & 0 \\
0 & M_{s}
\end{array}\right]\left[\begin{array}{c}
\ddot{z}_{i} \\
\ddot{z}_{s i}
\end{array}\right]+\left[\begin{array}{cc}
b_{r} & -b_{r} \\
-b_{r} & b_{r}+b_{s}
\end{array}\right]\left[\begin{array}{c}
\dot{z}_{i} \\
\dot{z}_{s i}
\end{array}\right]+\left[\begin{array}{cc}
k_{r} & -k_{r} \\
-k_{r} & k_{r}+k_{s}
\end{array}\right]\left[\begin{array}{c}
z_{i} \\
z_{s i}
\end{array}\right]=\left[\begin{array}{c}
F(t) \\
0
\end{array}\right],
$$

where $i=1,2, \ldots, N$ with $N$ denoting the total number of sleepers considered in the model.

a)
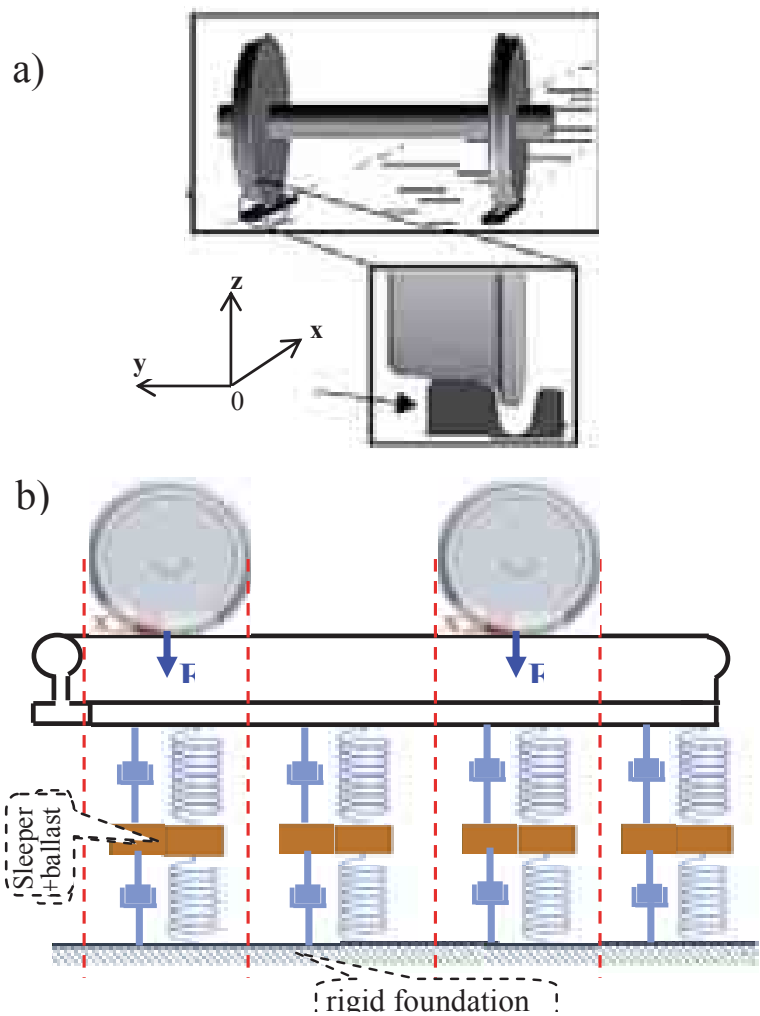

c)

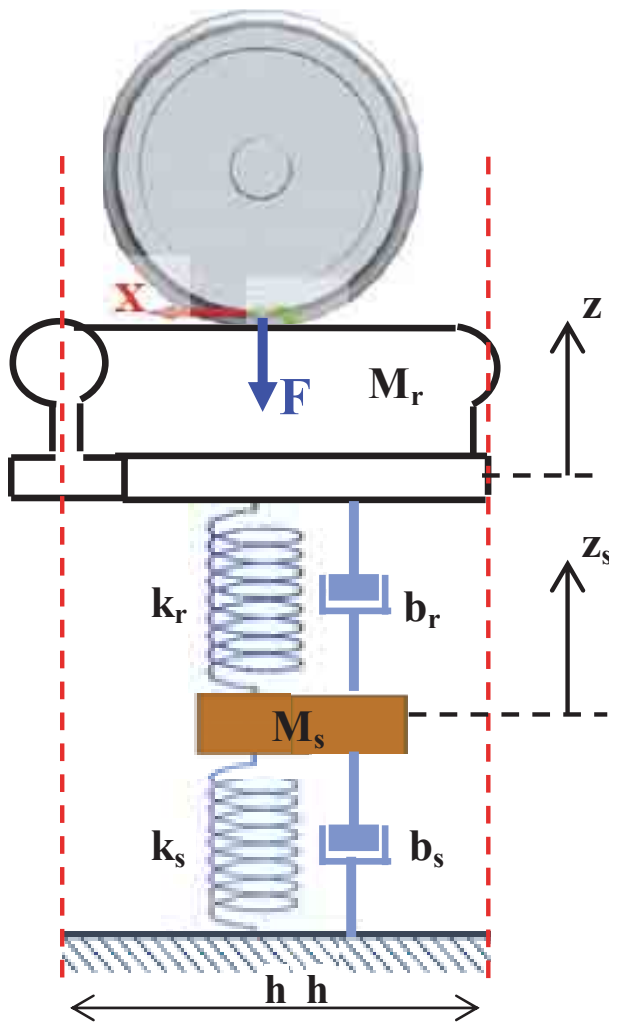

Fig. 5. Model of the wheel-rail pair: a) wheel-rail contact, b) subdivision into segments, c) one segment

The motion of the rail beam coupled with the sleeper and ballast is expressed as $\mathrm{z}_{\mathrm{i}}=\mathrm{z}\left(y_{i}, t\right)$, while $z_{s i}=z_{s i}(t)$ describes the motions of the sleeper and ballast masses. Parameters: $k_{p}, k_{s}, b_{r}$ and $b_{s}$ are the rail and ballast stiffness and damping coefficients, respectively. The rail mass per segment is represented by $M_{r}$, and $M_{s}$ is the mass of the half of sleeper and ballast block together.

The system vibrations are excited by the loading force $F(t)$ exhibiting variations in time shown in Fig. 6a. It is worth noticing that the short duration pulses correspond to small dimensions of the contact areas. The duration and intensities of these pulses depend on the vehicle mass and the speed of the train. The idealized pulsed supplying force $F(t)$ (Fig.6b) can be represented by more convenient formula when introducing the unit Heaviside functions $H_{k}\left(t, T_{k}\right)$, namely:

$$
F(t)=A+\sum_{k=1}^{3} H_{k}\left(t, T_{k}\right)\left[\delta_{k, 2} \cdot A\right],
$$

where $\delta_{k, 2}$ denotes the Kronecker delta operator.

In what follows we focus the attention on one segment only and a multi segment system can be considered by appropriate extension of the presented approach. Applying the algorithm presented in the previous section we can express the resulting steady state coordinate $\mathrm{z}(\mathrm{t})$ as follows:

$$
z(t)=z(t+T)=z_{1}(t)+\sum_{k=1}^{3} H\left(t, T_{k}\right)\left[z_{k+1}(t)-z_{k}(t)\right],
$$

where $\mathrm{z}_{\mathrm{k}}(t), k=1,2,3,4$ denote the coordinates excited by the parts of the supplying force period. 
a)

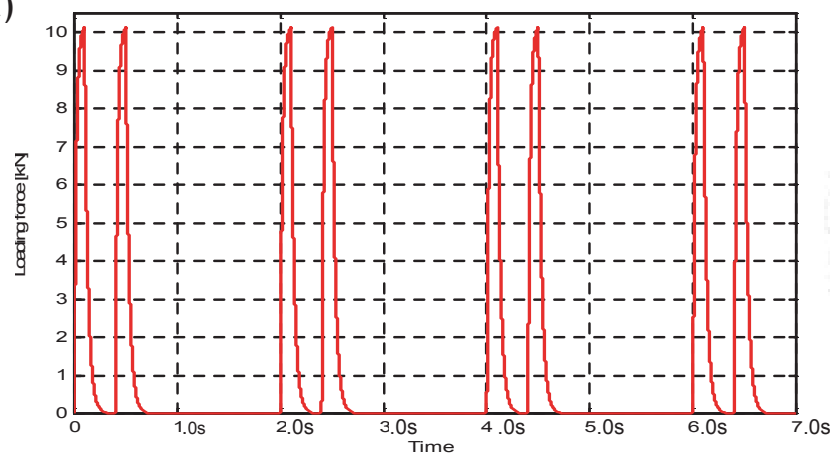

b)

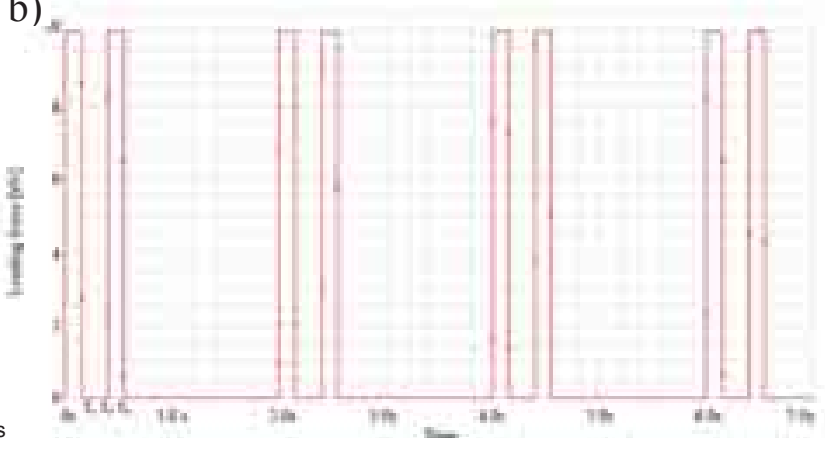

Fig. 6. Variations in time of the loading force pulses: a) real, b) ideal

When $t \in\left(T_{k}, T_{k+1}\right)$ and the supplying force is equal to $A$ or 0 with $k=1,2,3$, 4 we obtain:

$$
z_{k}(t)=\sum_{m=1}^{4} B_{m, k} e^{s_{m} t}+\frac{k_{1}+k_{2}}{k_{1} k_{2}} \delta_{k, 2 l+1} \cdot A,
$$

with $l=0$ for $k=1$ and 2 and $l=1$ for $k=3$ and 4 . Constants $B_{m, k}, m=1,2,3,4$, are to be determined from continuity and periodicity conditions.
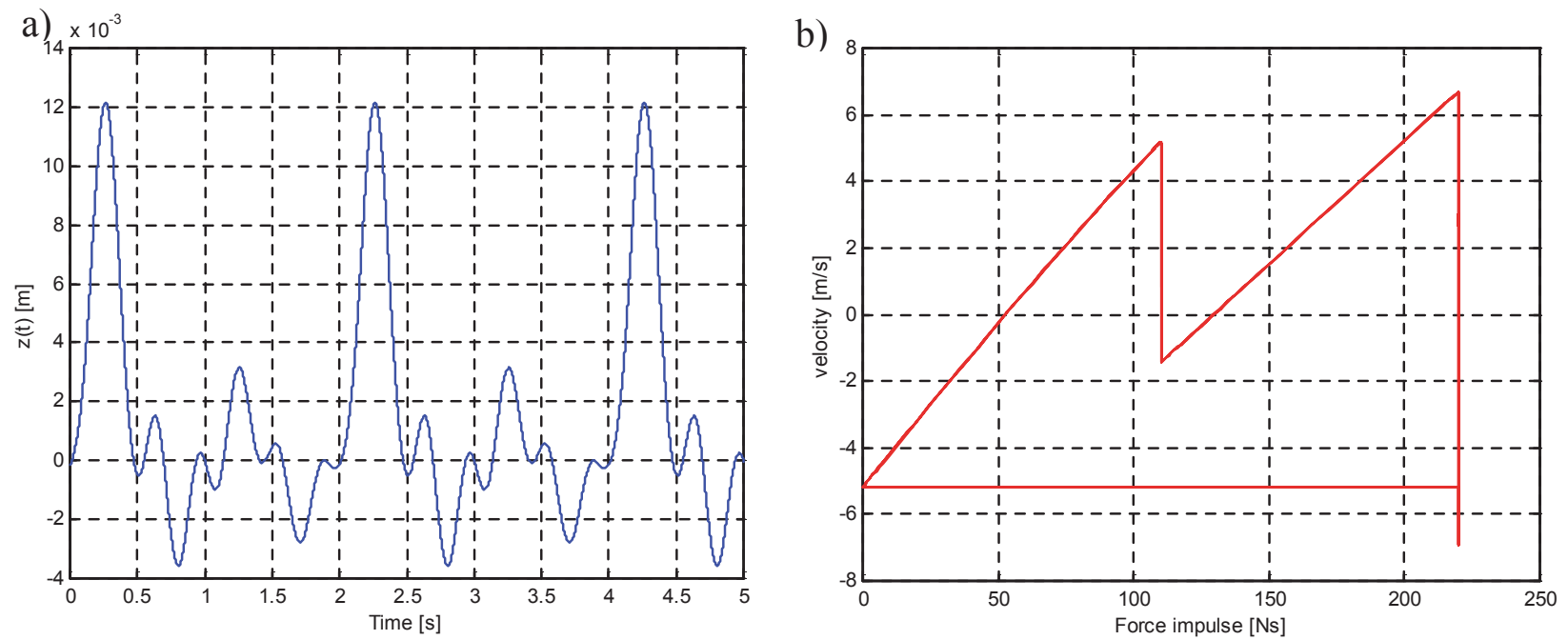

Fig. 7. Simulation results: a) rail segment displacement, b) hysteretic loop of one period energy

The solution has been easily determined by using the carrying periodic waveform $p(t)$ and concatenation procedure in accord to (21). Performed computer simulations for a set of the system parameters: $A=110 \mathrm{kN}, T_{1}=0.1 \mathrm{~s}, T_{2}=0.4 \mathrm{~s}, T_{3}=0.5 \mathrm{~s}, T=2 \mathrm{~s}$ and $M_{r}=110 \mathrm{~kg}, M_{s}=520 \mathrm{~kg}, k_{r}=250$ $\mathrm{N} / \mathrm{m}, k_{s}=150 \mathrm{~N} / \mathrm{m}, b_{r}=632 \mathrm{Ns} / \mathrm{m}$ and $b_{s}=520 \mathrm{Ns} / \mathrm{m}$ have yielded variations in time of the rail segment displacement presented in Fig. 7 a.

The presented methodology can be applied to predict the energy transferred from the wheel the rail segments during one period of the forcing term. In the case of the above considered systems parameters the one-period energy absorbed by the rail segment is expressed as an area encompassed by the cyclic trajectory on the energy phase plane. It is presented in Fig. $7 \mathrm{~b}$.

\section{Hysteretic loops of one-period energy}

The emphasis in this section is put on the somewhat complementary aspect of shaping the energy of elements in a track system, which directly involves the hysteretic loop in the energy phase plane, as opposed to the widely up-to-date used concept of the active, reactive and distortion 
powers. As is well known, all up-to-date used methods for energy determination of a system element operating in a periodic non-harmonic steady state have many insufficiencies, which vary from one case to other and importantly depend on the assumed interpretation of particular components of the apparent power defined in the complex number domain [4].

Under periodic conditions the total energy $\Delta W(n T)$ delivered by a force source to its load is measured as $n$ multiple of the area $W_{T}$ of one period energy loop determined on the energy phase plane. Thus we have:

$$
\Delta W(t)_{\mid t=n T}=n W_{T},
$$

where the energy $W_{T}$ delivered by supplying force $F(t)=F(t+T)$ to the load during one period equals:

$$
W_{T}=\int_{0}^{T} F(t) v(t) d t=\int_{z(0)}^{z(T)} F(t) d z(t)=\int_{P(0)}^{P(T)} v(t) d P(t),
$$

with $v(t)$ and $P(t)=\int_{0}^{t} F(\tau) d \tau$ denoting the velocity of the rail displacement and the force impulse.
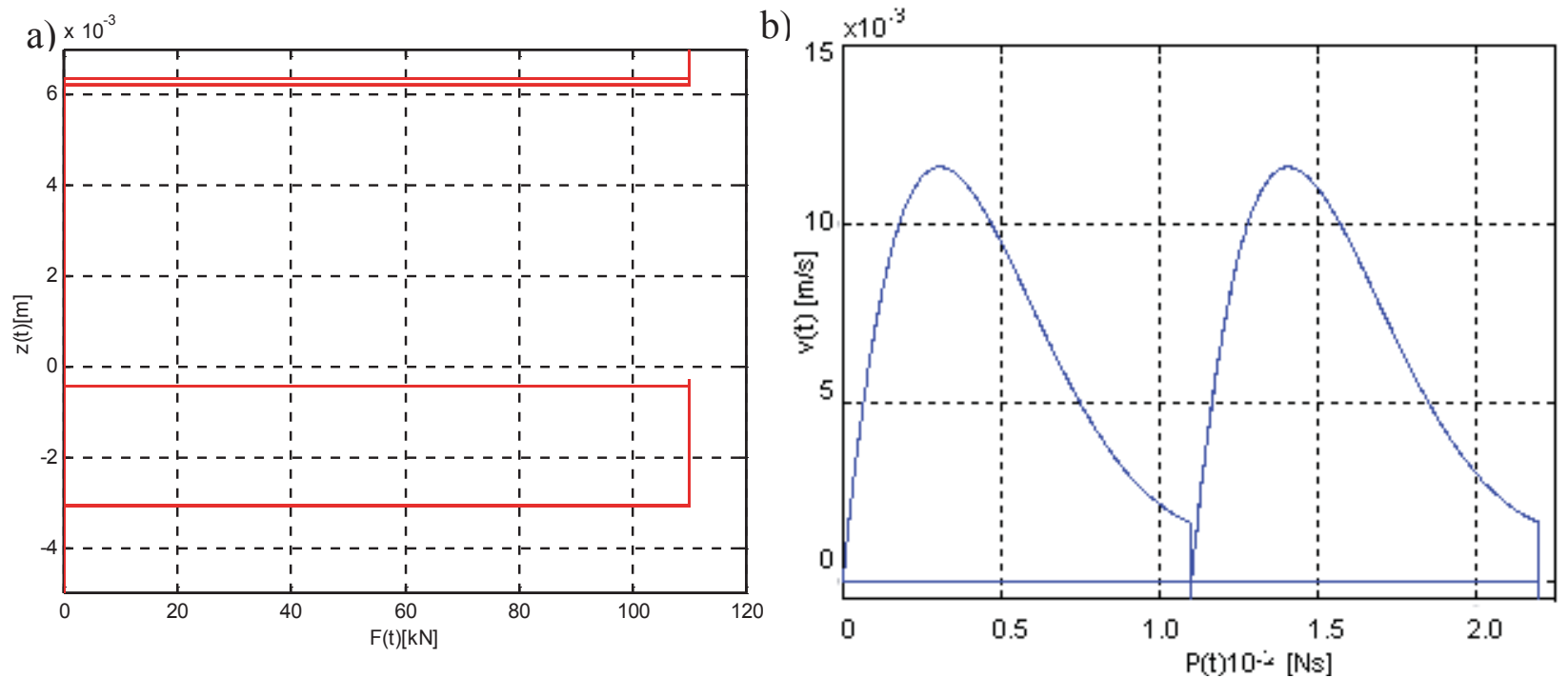

Fig. 8. One-period energy loops for discontinuous loads: a) two segments, b) sleeper nonlinear model

The above integral is of the Riemann-Stieltjes type and admits integration by parts in the form

$$
W_{T}=\int_{z(0)}^{z(T)} F(t) d z(t)=F(T) z(T)-F(0) z(0)-\int_{0}^{T} z(t) \dot{F}(t) d t,
$$

where $\dot{F}(t)$ denotes the time derivative.

The important point to note here is a common feature of the energy hysteretic loops lying in that that the properties of this integral can be used to prove many identities concerning the analysis of the one-period energy, in particular, when the periodic forcing waveform $F(t)$ exhibits discontinuities. Moreover, it follows from expression (24) that the area enclosed by a loop on the energy phase-plane with coordinates $(F(t), z(t))$ or, equivalently, $(v(t), P(t))$ determines the oneperiod energy $W_{T}$ because in the periodic state all system waveforms exhibit the same period. Illustrations are presented in Fig.8a. The above-established model of the wheel-rail contact dynamics has been applied to that same roll plane but with taking into account a nonlinear characteristic of the sleeper with respect the ground. It is represented by the relation:

$$
f_{2}\left(z_{s}\right)=k_{2} \cdot\left(\alpha \cdot z_{s}^{2}+\beta \cdot z_{s}^{3}\right),
$$


where constant parameters $k_{2}, \alpha$ and $\beta$ can be considered as bifurcating values. For $\alpha=1.75, \beta=-1$ $k_{2}=1.5 \mathrm{kN} / \mathrm{m}$ and other parameters as those considered in the case of Fig. 8a the determined loop of one period energy is shown in Fig. 8b. Thus, for any system operating in periodic non-harmonic conditions it is advantageous to produce directly the one-period energy $W_{T}$ without recourse to any forms neither of its power nor the Fourier series approach. The importance of the one-period energy loop lies mainly in the fact that it can be used to establish many useful expressions concerning the influence of input waveform shapes, parameter values and structures of systems on the quality of the energy delivered to loads.

\section{Material behaviour in wheel-rail contact}

The behaviour of wheel and rail materials under wheel-rail contact involves the following deterioration mechanisms: wear, plastic deformation and rolling contact fatigue [9]. The basic properties, which must have rails, are high abrasion resistance and flexural strength, and the corresponding hardness and elasticity. Therefore, to produce them used is the right stainless steel called a rail steel made of pearlitic steel. The main components of rail steel, in addition to $\mathrm{Fe}$ and
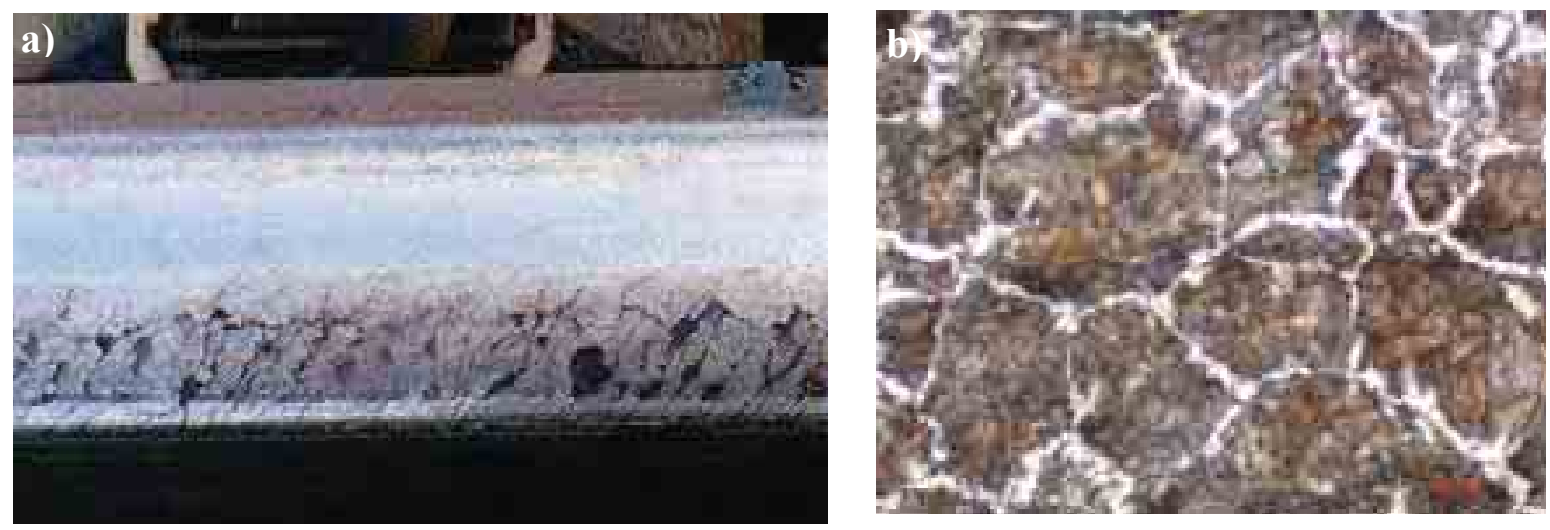

Fig. 9. Images of a train rail: a) surface with characteristic undulations, b) defects in material crystalline structure

carbon $\mathrm{C}(0.6-0.82 \%)$, there are various elements such as manganese, Mn (0.6 to 2.1\%), silicon Si $(0.1-0.90 \%)$, chromium $\mathrm{Cr}(\leq 1.3 \%)$, phosphorus $(\leq 0.05 \%)$, sulphur $(\leq 0.05 \%)$ and others that improve the mechanical properties of the resulting material. On surfaces of railway rail heads and wheels it is easily seen under an optical microscope the surface layer with a thickness of 10 to $100 \mu \mathrm{m}$, which is white in colour after etching in a solution containing mainly nitric acid. It is called white layer (WEL) and has a significant impact on the life of the rails and wheels. When the thickness of WEL exceeds $50 \mu \mathrm{m}$, formation of cracks leading to chipping takes places (Fig.9a).

The identification of the energy delivered by the load to the rail and the role of both material properties and the rail-wheel contact conditions is critical in achieving improved component performance. Is also growing need to find a steel with a higher microstructural stability, allowing for longer life. Depending on the applied loads, the depth of deformation can be anything from a few microns to $5-10 \mathrm{~mm}$. Temperatures above $700 \mathrm{C}$ at the interface between wheel and rail lead to the transformation of pearlite to austenite. Austenitizing temperature of head rails is also lowered, and much, through the high density of crystalline defects (Fig. 9b). Rapid cooling of austenite leads to the formation of tetragonal-martensite supersaturated solution carbon in the iron. Studies show that in the formation of WEL grain size is significantly reduced and the formation of corrugated places is not a process of high temperature but is caused only by plastic deformation $[2,11]$.

\section{Summary and conclusions}

Theoretical models describing the interaction between a railway wheel and the track rail were used for studying the rail vibrations. The main emphasis has been put on one-period energy 
delivered to the rail by the loading force exerted by the railway vehicle. It has been shown that the time domain representation of a system by means of the concatenation procedure, as opposed to the frequency domain representation by means of the system transfer function, has become more advantageous approach to the exhibition of system dynamics in periodic non-harmonic states. Especially, the introduction hysteretic loops on energy phase plane greatly advances the theory and enables the proper generalization of many fundamental concepts known for computer aided geometric designs to the world of periodic non-harmonic waveforms.

\section{References}

[1] Bezin, Y., et al., The effect of dynamic rail roll on the wheel-rail contact conditions, Vehicle System Dynamics, Vol. 46, pp. 107- 117, 2008.

[2] Chudzikiewicz, A., Myśliński, A., On wheel-rail contact problem with material properties varying with depth, PAMM, Proc. Applied Mathematic Mechanics, Vol. 9, pp.721-722, 2009.

[3] Hayat, M.M., Understanding of Rail Wheel Fatigue: Rolling Contact Fatigue in Rail-wheel, Saarbrücken, LAP LAMBERT Academic Publishing, 2012.

[4] Haykin, S., Van Veen, B., Signals and Systems, John Wiley, New York 2003.

[5] Iwnicki, S., (ed.), Handbook of Railway Vehicle Dynamics, Boca Raton, Taylor\&Francis, 2006.

[6] Karnopp, D., Vehicle Stability, Marcel Dekker, Inc., New York 2004.

[7] Misiak, J., Stachura, S., Selected problems of static stability and dynamics of rod and shell structures, Publish. Office, Warsaw University of Ecology and Management, Warsaw 2010.

[8] Redfern, D., Campbell, C., The MATLAB ${ }^{\circledR} 5$ Handbook, Springer, New York 1998.

[9] Ren, L., et al., Properties of wheel/rail longitudinal creep force due to sinusoidal short pitch corrugation on railway rail, Wear, Vol. 284-285, pp. 73-81, 2012.

[10] Steenbergen, M. J., Modelling of wheels and rail discontinuities in dynamic wheel-rail contact analysis, Vehicle System Dynamics, Vol. 44, pp.763-787, 2006.

[11] Trzaska, M., Structure and properties of rail steel, Research report (in Polish), Warsaw University of Technology, Faculty of Materials Science and Engineering, Warsaw 2011.

[12] Trzaska, Z., Modelling of energy processes in wheel-rail contacts operating under influence of periodic discontinuous forces, Journal of Transportation Technologies,Vol.2, pp. 129-143, 2012.

[13] Zerzm, E., Topics in Multidimensional Linear Systems Theory, Springer, Berlin 2000. 